\title{
Decentralized Prediction of End-to-End Network Performance Classes
}

\author{
Yongjun Liao*, Wei Du†, Pierre Geurts ${ }^{\ddagger}$, and Guy Leduc* \\ ${ }^{*}$ Research Unit in Networking, University of Liège, Belgium \\ $\dagger$ Intelligent and Interactive Systems, University of Innsbruck, Austria \\ $\ddagger$ Systems and Modeling, University of Liège, Belgium
}

\begin{abstract}
In large-scale networks, full-mesh active probing of end-toend performance metrics is infeasible. Measuring a small set of pairs and predicting the others is more scalable. Under this framework, we formulate the prediction problem as matrix completion, whereby unknown entries of an incomplete matrix of pairwise measurements are to be predicted. This problem can be solved by matrix factorization because performance matrices have a low rank, thanks to the correlations among measurements. Moreover, its resolution can be fully decentralized without actually building matrices nor relying on special landmarks or central servers.

In this paper we demonstrate that this approach is also applicable when the performance values are not measured exactly, but are only known to belong to one among some predefined performance classes, such as "good" and "bad". Such classification-based formulation not only fulfills the requirements of many Internet applications but also reduces the measurement cost and enables a unified treatment of various performance metrics. We propose a decentralized approach based on Stochastic Gradient Descent to solve this class-based matrix completion problem. Experiments on various datasets, relative to two kinds of metrics, show the accuracy of the approach, its robustness against erroneous measurements and its usability on peer selection.
\end{abstract}

\section{Categories and Subject Descriptors}

C.2.4 [Computer Communication Networks]: Distributed Systems-Distributed Applications

\section{General Terms}

Measurement

Permission to make digital or hard copies of all or part of this work for personal or classroom use is granted without fee provided that copies are not made or distributed for profit or commercial advantage and that copies bear this notice and the full citation on the first page. To copy otherwise, to republish, to post on servers or to redistribute to lists, requires prior specific permission and/or a fee.

ACM CoNEXT 2011, December 6-9 2011, Tokyo, Japan.

Copyright 2011 ACM 978-1-4503-1041-3/11/0012 ...\$10.00.

\section{Keywords}

network performance classification, matrix completion, matrix factorization, stochastic gradient descent

\section{INTRODUCTION}

The knowledge of end-to-end network performance is essential for Internet applications to achieve Quality of Service (QoS) objectives [24, 17, 18, 27]. Typical QoS objectives relate to performance metrics such as network delay, often represented by round-trip time (RTT), available bandwidth (ABW) and packet loss rate (PLR) [6]. Under a chosen metric, the acquisition of end-to-end network performance amounts to perceiving measurements of some kind, commonly in the form of real-valued quantities.

While such quantitative measures have been widely accepted by the networking community, qualitative measures of whether the performance of a network path is good enough already carry information of great interest from the application point of view. For instance, streaming media applications care more about whether the ABW of a path is high enough to provide smooth playback quality. In peer-to-peer applications, although finding the nearest nodes to communicate with is preferable, it is often enough to access a nearby node with a limited loss compared to the nearest node. Moreover, qualitative measures have two nice properties. First, they are generally coarse and can therefore be acquired with cheaper means. The cost reduction is more significant for metrics such as the ABW whose measurement is expensive. Second, qualitative measures are stable and better reflect long-term characteristics of network paths, which means that they can be probed less often.

In this paper, we consider the inference of whether the performance of a network path is good enough as a binary classification problem [2] that classifies the performance, according to a metric, into one of the two classes of "good" and "bad" 1 . Such class-based representation

\footnotetext{
${ }^{1}$ Depending on the context, the class labels of "good" and "bad" may refer to "well-performing" and "poorlyperforming" or "well-connected" and "poorly-connected".
} 
enables a unified and equal treatment of different metrics such as $\mathrm{RTT}$ and $\mathrm{ABW}$, which have vastly distinct characteristics and are usually discriminatively treated.

A major obstacle to the utilization of end-to-end network performance by Internet applications is the scalable acquisition in large-scale networks. As full-mesh active probing among all nodes is obviously infeasible, a natural idea is to probe a small set of pairs and then predict the performance between other pairs where there are no direct measurements, which has been successfully used to estimate RTTs in various Network Coordinate Systems (NCS) [8].

Under the "probe a few and predict many" framework, we investigate the prediction of end-to-end network performance classes in large-scale networks. In contrast to previous work, the prediction of network performance is formulated as a matrix completion problem where a partially observed matrix is to be completed [5]. Here, the matrix contains binary performance measures between network nodes with some of them known and the others unknown, and thus to be filled. Matrix completion is only possible if matrix entries are largely correlated, which holds for many metrics in the Internet because Internet paths with nearby end nodes often overlap and share common bottleneck links. These redundancies among network paths cause the constructed performance matrix to be low rank (we will demonstrate this empirically for RTT and ABW).

The low-rank nature of various performance matrices enables their completion by matrix factorization techniques. This paper presents a novel decentralized matrix factorization approach based on Stochastic Gradient Descent (SGD). By letting network nodes exchange messages with each other, the factorization is collaboratively and iteratively performed at all nodes with each node equally retrieving a small number of measurements. A distinct feature of our approach is that it is fully decentralized, with neither explicit construction of matrices nor special nodes such as landmarks or a central node where measurements are collected and processed. Extensive experiments on various RTT and ABW datasets show that our approach is simple, suitable for dealing with dynamic measurements in largescale networks and robust against large amount of erroneous measurements. Furthermore, we demonstrate the benefits of our binary classification approach on peer selection, a problem at the heart of many Internet applications. These experiments highlight not only the accuracy and the applicability of our approach but also its advantage in terms of measurement cost reduction.

The rest of the paper is organized as follows. Section 2 describes related work. Section 3 introduces the metrics and the classification of end-to-end network performance. Section 4 gives the formulation of network performance prediction as matrix completion and its resolution by low-rank matrix factorization. Section 5 describes the decentralized matrix factorization algorithms based on Stochastic Gradient Descent. Sections 6 experiments our approach on three publicly available datasets of RTT and of ABW. Conclusions and future work are given in Section 7.

\section{RELATED WORK}

Most related work on network performance prediction focused on the prediction of real values of some performance metric with a particular interest for the RTT. Examples include Global Network Positioning [14], Vivaldi [7], Internet Distance Estimation Service [13] and Decentralized Matrix Factorization [12]. The prediction of ABW is less successful with SEQUOIA the only work found in the literature that embedded ABW measurements into a tree metric [16]. On predicting performance classes, [20] is to our knowledge the only related work that used an advanced machine learning technique, namely max-margin matrix factorization (MMMF) [22]. Although successful especially thanks to the incorporation of active sampling, MMMF required a semidefinite programming solver that only works for smallscale problems and in a centralized manner.

The main contributions of our work are the treatment of network performance as classes, instead of real values, and the formulation of the class prediction problem as matrix completion. Although numerous approaches to matrix completion have been proposed, few of them are designed for network applications where decentralized processing of data is appreciated. Thus, we developed a fully decentralized approach based on Stochastic Gradient Descent (SGD) which is founded on the stochastic optimization theory with nice convergence guarantees [3]. Two slightly different algorithms are proposed for dealing with RTT and with ABW due to their different measurement methodology. We then studied empirically the sensitivity of the algorithms to the parameters, its robustness against erroneous measurements and its applicability in Internet applications.

\section{METRICS AND CLASSIFICATION OF NETWORK PERFORMANCE}

\subsection{Metrics of Network Performance}

While ultimately the performance of a network path should be judged by the QoS perceived by end users, it is important to define an objective metric that is directly measurable without users' interventions. Among the commonly-used metrics mentioned earlier, this paper only discusses RTT and ABW due to the availability of the data and the relatively rare occurrence of packets losses in the Internet [6].

\subsubsection{Round-Trip Time (RTT)}


The RTT is one of the oldest and most commonlyused network measurements due partially to its ease of acquisition by using ping with little measurement overhead, i.e., few ICMP echo request and response packets between the sender and the target node. Although the one-way forward and reverse delays are not exactly the same due to e.g. the routing policy and the Internet infrastructure, the RTTs between two network nodes can approximately be treated as symmetric.

\subsubsection{Available Bandwidth (ABW)}

The ABW is the available bandwidth of the bottleneck link on a path. A comparative study has shown that tools based on self-induced congestion are generally more accurate [21]. The idea is that if the probing rate exceeds the available bandwidth over the path, then the probe packets become queued at some router, resulting in an increased transfer time. The ABW can then be estimated as the minimum probing rate that creates congestions or queuing delays. To this end, pathload [10] sends trains of UDP packets at a constant rate and adjusts the rate from train to train until congestions are observed at the target node. Pathchirp [19] reduces the probe traffic by varying the probe rate within a train exponentially.

Compared to RTT, measuring ABW is much more costly and less accurate, suffering from an underestimation bias due to the bursts of network traffic and the presence of multiple links with roughly the same ABW. This bias can be relieved by increasing the probe packet train length at the cost of more measurement overhead [6]. In contrast to the RTT which is inferred by the sender, the ABW is clearly asymmetric and its measurement is inferred at the target node.

\subsection{Classification of Performance Metrics}

The classification of network performance amounts to determining some qualitative measure that reflects how well a path can perform according to a metric. In this paper, we focus on the classification of network performance into two classes of "good" and "bad", represented by 1 and -1 respectively, independently from the actual metric used.

A straightforward approach to classifying network performance is by thresholding, i.e., comparing the value of the metric with a classification threshold, denoted by $\tau$, which is determined to meet the requirements of the applications. For example, Google TV requires a broadband speed of $2.5 \mathrm{Mbps}$ or more for streaming movies and 10Mbps for High Definition contents [9]. Accordingly, $\tau=2.5 \mathrm{Mbps}$ or $10 \mathrm{Mbps}$ can be defined for $\mathrm{ABW}$ to separate "good" paths from "bad" ones. The impact of $\tau$ will be demonstrated in Section 6 .

Classification by thresholding is directly applicable to
RTTs whose measurements are cheap. For the ABW, it is preferable to directly obtain the class measures without the explicit acquisition of the values in order to reduce measurement overhead. This is plausible for tools based on self-induced congestion as each probe by sending a UDP train at a constant rate naturally yields a binary response of "yes" or "no" that suggests whether the $\mathrm{ABW}$ is larger or smaller than the probe rate. Thus, the direct classification of $\mathrm{ABW}$ is trivial and can be done with existing tools such as pathload and pathchirp with little modification:

pathload Send UDP trains at a constant rate of $\tau$, and classify the path as "good" if no congestion is observed and "bad" otherwise.

pathchirp Use pathchirp with fewer and shorter probe trains, and threshold by $\tau$ the rough and inaccurate quantities obtained.

The directly measured performance classes may be inaccurate especially for those paths with metric quantities close to $\tau$. We will demonstrate the impact of the inaccuracy of the class measures in Section 6 .

The classification of other performance metrics can be done similarly by exploiting their different nature and different measurement techniques. As generally data acquisition undergoes the accuracy-versus-cost dilemma that accuracy always comes at a cost, coarse measures of performance classes are always cheaper to obtain than exact quantities, regardless of the metric used.

\section{NETWORK PERFORMANCE PREDIC- TION BY MATRIX FACTORIZATION}

\subsection{Formulation as Matrix Completion}

The problem of network performance prediction can generally be formulated as a matrix completion problem. Specifically, assuming $n$ nodes in a network, given a partially observed $n \times n$ matrix $X$ containing performance measures in the form of either real-valued quantity or discrete-valued class, a matrix of the same size $\hat{X}$ needs to be found that best predict the unobserved entries of $X$. Denote $x_{i j}$ the true performance measure from node $i$ to node $j$ and $\hat{x}_{i j}$ the predicted measure. We refer to the estimation of discrete-valued classes as class-based prediction and the estimation of real-valued quantities as quantity-based prediction.

A common approach to matrix completion is low-rank approximation, which minimizes the following function

$$
\begin{aligned}
L(X, \hat{X}, W)= & \sum_{i, j=1}^{n} w_{i j} l\left(x_{i j}, \hat{x}_{i j}\right), \\
& \text { subject to } \operatorname{Rank}(\hat{X})=r \ll n .
\end{aligned}
$$

where $W$ is a weight matrix with $w_{i j}=1$ if $x_{i j}$ is known and 0 otherwise. $l$ is a loss function that penalizes the 


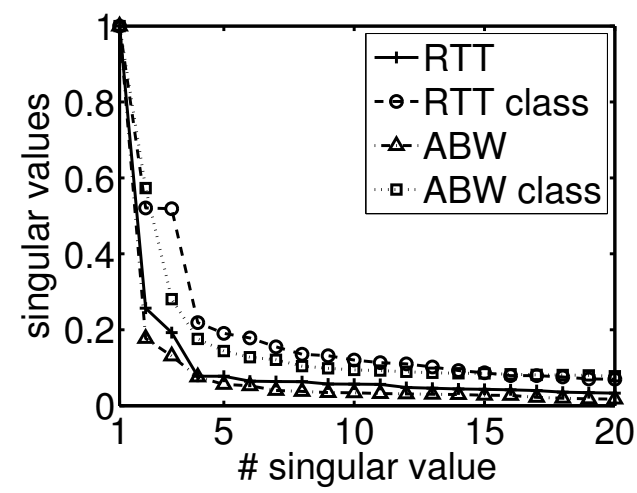

Figure 1: The singular values of a RTT and a ABW matrix and of their binary class matrices. The RTT matrix is $2255 \times 2255$ and extracted from the Meridian dataset [25]. The ABW matrix is $201 \times 201$ and extracted from the HP-S3 dataset [26]. The binary class matrices are obtained by thresholding their corresponding measurement matrices with $\tau$ equal to the median value of each dataset. The singular values are normalized so that the largest singular values of all matrices are equal to 1 .

difference between an estimate and its desired or true value. For predicting real-valued quantities, the $L_{2}$ or square loss function is commonly used. For predicting discrete-valued classes, more often used are the hinge and the logistic loss functions [2]. These different loss functions are given below.

- $L_{2}$ or square loss function: $l(x, \hat{x})=(x-\hat{x})^{2}$;

- hinge loss function: $l(x, \hat{x})=\max (0,1-x \hat{x})$;

- logistic loss function: $l(x, \hat{x})=\ln \left(1+e^{-x \hat{x}}\right)$.

Note that the hinge loss function is not differentiable.

In the above loss functions, $x$ is the reference value and $\hat{x}$ is the predicted value. In the setting of binary classification, $x$ is either 1 or -1 and $\hat{x}$ is typically real-valued and its sign usually represents the predicted class. Both the hinge and logistic loss functions are such that values of $x \hat{x}$ lower than 1 are strongly penalized and otherwise less or not penalized. These classification loss functions are thus not sensitive to the actual value of $\hat{x}$ as long as its sign matches the sign of $x$. This contrasts to the $L_{2}$ loss function which has smaller penalties when $\hat{x}$ approaches to $x$.

The assumption in this low-rank approximation is that the entries of $D$ are largely correlated, which causes $D$ to have a low effective rank. To show that it holds for our problem, Figure 1 plots the singular values of a RTT and a ABW matrix and of their binary class matrices. It can be seen that the singular values of all matrices decrease fast, indicating strong correlations in both RTT and ABW measurements. The low-rank nature of many other RTT datasets have been previously reported in [23].

\subsection{Low-Rank Matrix Factorization}

Directly minimizing eq. 1 is difficult due to the rank constraint. However, as $\hat{X}$ is of rank $r$, we can factorize it into the product of two smaller matrices,

$$
\hat{X}=U V^{T},
$$

where $U$ and $V$ have $r$ columns. Therefore, we can look for $(U, V)$ instead by minimizing

$$
\begin{aligned}
& L(X, U, V, W, \lambda)= \\
& \quad \sum_{i, j=1}^{n} w_{i j} l\left(x_{i j}, u_{i} v_{j}^{T}\right)+\lambda \sum_{i=1}^{n} u_{i} u_{i}^{T}+\lambda \sum_{i=1}^{n} v_{i} v_{i}^{T},
\end{aligned}
$$

where $u_{i}$ and $v_{i}$ are the $i$ th rows of $U$ and $V$ respectively, and $u_{i} v_{j}^{T}=\hat{x}_{i j}$ is the estimate of $x_{i j} . \lambda$ is the regularization coefficient that controls the extent of the regularization, the incorporation of which is to overcome a well-known problem called overfitting in the field of machine learning [2]. In words, directly minimizing eq. 3 often leads to a "perfect" model with no or little errors on the training data (i.e., the known entries) while having large errors on the unseen data (i.e., the unknown entries) which are not used in learning.

The class of techniques to minimize eq. 3 is matrix factorization. In the presence of missing entries or in case of loss functions other than the $L_{2}$ loss, eq. 3 becomes non-convex and a local optimal solution can be found by iterative optimization methods such as Gradient Descent and Newton algorithms [4]. Note that matrix factorization has no unique solutions as

$$
\hat{X}=U V^{T}=U G G^{-1} V^{T},
$$

where $G$ is any arbitrary invertible matrix. Therefore, replacing $U$ by $U G$ and $V^{T}$ by $G^{-1} V^{T}$ will not change the approximation error.

\subsection{System Architecture}

The above low-rank matrix factorization is generic to predict network performance of all kinds, by all metrics and in the form of either quantity or class. In this paper, we focus on binary classification where $x_{i j}$ in $X$ is either 1 or -1 , representing "good" and "bad".

Figure 2 illustrates a unified architecture that consists of a measurement module and a prediction module. The measurement module probes the performance classes of a small number of paths and put them in the corresponding entries of a large matrix. The prediction module estimates the missing entries by applying a matrix factorization technique. The estimates of the missing entries are typically real-valued and the corresponding predicted classes can be determined by e.g. taking the sign of $\hat{x}_{i j}$. 


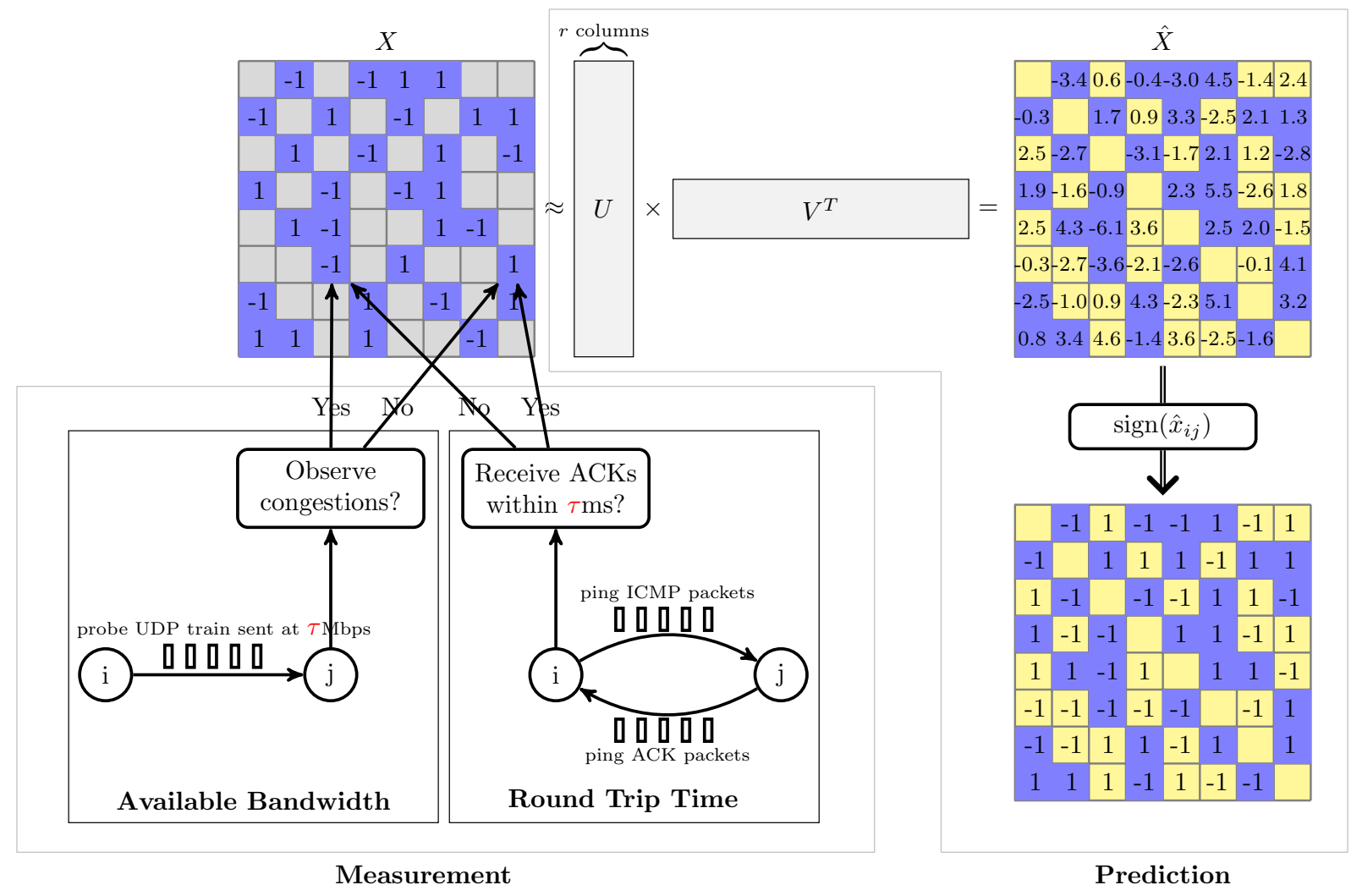

Figure 2: Architecture of class-based network performance measurement and prediction. Note that the diagonal entries of $X$ and $\hat{X}$ are empty.

\section{DECENTRALIZED MATRIX FACTORIZATION BY STOCHASTIC GRADIENT DESCENT}

The above system has a centralized architecture that requires the collection and the processing of the measurements at a central node. This section introduces a fully decentralized matrix factorization approach based on a particular stochastic optimization method, namely Stochastic Gradient Descent (SGD).

\subsection{Stochastic Gradient Descent (SGD)}

SGD is one of stochastic optimization methods that has been widely used for on-line and large-scale machine learning [3]. It is a variation of traditional Batch Gradient Descent. However, instead of collecting all training samples beforehand and computing the gradients over them, each iteration of SGD chooses one training sample at random and updates the parameters being estimated along the negative gradients computed over that chosen sample. Averaging each SGD update over all possible choices of the training samples would restore the batch gradient descent [3].

SGD is particularly suitable for solving the problem of network performance prediction, as measurements can be acquired on demand and processed locally with no need to store them. It also has simple update rules that involve only vector operations and is able to deal with large-scale dynamic network measurements.

\subsection{SGD for Network Performance Prediction}

As a decentralized optimization of eq. 3 forbids the explicit constructions of any matrices, each row of $U$ and of $V, u_{i}$ and $v_{i}$, are stored distributively at each node of the network. In the sequel, $u_{i}$ and $v_{i}$ will be called the coordinates of node $i$. To calculate $u_{i}$ and $v_{i}$, $i=1, \ldots, n$, only local measurements related to node $i$ are needed.

Due to their different measurement methodology, RTT and $\mathrm{ABW}$ are treated slightly differently.

\subsubsection{SGD for Dealing with RTT}

RTT is symmetric and is probed and inferred by the sender. Thus, when a RTT measurement $x_{i j}$ is acquired and available at node $i, u_{i}$ of node $i$ can be immediately updated by using $x_{i j}$. As $x_{i j}=x_{j i}$ for RTT, $x_{i j}$ can also be used by node $i$ to update $v_{i}$.

Hence, given $x_{i j}$, the errors to be reduced at node $i$ are

$$
\begin{gathered}
E_{i j}=l\left(x_{i j}, u_{i} v_{j}^{T}\right)+\lambda u_{i} u_{i}^{T}, \\
E^{i j}=l\left(x_{i j}, u_{j} v_{i}^{T}\right)+\lambda v_{i} v_{i}^{T} .
\end{gathered}
$$


The gradients, by ignoring the non-differentiability of some loss functions, are given by

$$
\begin{aligned}
& \frac{\mathrm{d} E_{i j}}{d u_{i}}=\frac{\mathrm{d} l\left(x_{i j}, u_{i} v_{j}^{T}\right)}{\mathrm{d} u_{i}}+\lambda u_{i}, \\
& \frac{\mathrm{d} E^{i j}}{d v_{i}}=\frac{\mathrm{d} l\left(x_{i j}, u_{j} v_{i}^{T}\right)}{\mathrm{d} v_{i}}+\lambda v_{i},
\end{aligned}
$$

Note that we drop the factor of 2 , from the derivatives of the regularization terms and of the $L_{2}$ loss function below, for mathematical convenience.

The update rules of $u_{i}$ and $v_{i}$ are

$$
\begin{aligned}
& u_{i}=(1-\eta \lambda) u_{i}-\eta \frac{\mathrm{d} l\left(x_{i j}, u_{i} v_{j}^{T}\right)}{\mathrm{d} u_{i}}, \\
& v_{i}=(1-\eta \lambda) v_{i}-\eta \frac{\mathrm{d} l\left(x_{i j}, u_{j} v_{i}^{T}\right)}{\mathrm{d} v_{i}} .
\end{aligned}
$$

where $\eta$ is a learning rate.

\subsubsection{SGD for Dealing with $A B W$}

Different from RTT, ABW is asymmetric and is probed by the sender but inferred by the target node. Thus, when a ABW measurement $x_{i j}$ is available at node $j$, node $j$ needs to send $x_{i j}$ to node $i$ so that $u_{i}$ of node $i$ and $v_{j}$ of node $j$ can both be updated by using $x_{i j}$.

Hence, given $x_{i j}$, the error to be reduced at node $i$ and at node $j$ is

$$
E_{i j}=l\left(x_{i j}, u_{i} v_{j}^{T}\right)+\lambda u_{i} u_{i}^{T}+\lambda v_{j} v_{j}^{T} .
$$

With the gradients similarly defined as above, the update rules of $u_{i}$ and $v_{j}$ are

$$
\begin{aligned}
& u_{i}=(1-\eta \lambda) u_{i}-\eta \frac{\mathrm{d} l\left(x_{i j}, u_{i} v_{j}^{T}\right)}{\mathrm{d} u_{i}}, \\
& v_{j}=(1-\eta \lambda) v_{j}-\eta \frac{\mathrm{d} l\left(x_{i j}, u_{i} v_{j}^{T}\right)}{\mathrm{d} v_{j}} .
\end{aligned}
$$

\subsubsection{Gradients of Loss Functions}

For classification, the hinge and the logistic loss functions are the most commonly-used and adopted here. The gradients are given below. Without causing any confusion, the subscripts are dropped.

- For the hinge loss function, the gradients ${ }^{2}$ are zeros for correctly classified samples, i.e., those of $1-$ $x u v^{T} \leqslant 0$, and otherwise

$$
\begin{aligned}
& \frac{\mathrm{d} l\left(x, u v^{T}\right)}{\mathrm{d} u}=-x v, \\
& \frac{\mathrm{d} l\left(x, u v^{T}\right)}{\mathrm{d} v}=-x u,
\end{aligned}
$$

\footnotetext{
${ }^{2}$ As the hinge loss function is not differentiable, the gradient does not exist and is approximated by the subgradient [1]. However, we will only use the term gradient and gradient descent in this paper, following the convention in [3].
}

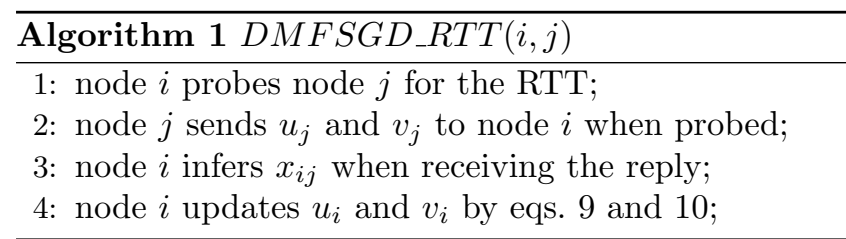

\begin{tabular}{l}
$\overline{\text { Algorithm } 2 D M F S G D \_A B W(i, j)}$ \\
\hline 1: node $i$ probes node $j$ for the ABW and sends $u_{i} ;$ \\
2: node $j$ infers $x_{i j}$ when probed; \\
3: node $j$ sends $x_{i j}$ and $v_{j}$ to node $i ;$ \\
4: node $j$ updates $v_{j}$ by eq. $13 ;$ \\
5: node $i$ updates $u_{i}$ by eq. 12 when receiving the reply;
\end{tabular}

- For the logistic loss function,

$$
\begin{aligned}
& \frac{\mathrm{d} l\left(x, u v^{T}\right)}{\mathrm{d} u}=-\frac{x v}{1+e^{x u v^{T}},} \\
& \frac{\mathrm{d} l\left(x, u v^{T}\right)}{\mathrm{d} v}=-\frac{x u}{1+e^{x u v^{T}}},
\end{aligned}
$$

The gradients of the $L_{2}$ loss functions for regression are also given here,

$$
\begin{aligned}
& \frac{\mathrm{d} l\left(x, u v^{T}\right)}{\mathrm{d} u}=-\left(x-u v^{T}\right) v, \\
& \frac{\mathrm{d} l\left(x, u v^{T}\right)}{\mathrm{d} v}=-\left(x-u v^{T}\right) u,
\end{aligned}
$$

as they will be used in Section 6 for the purpose of comparison of class-based and quantity-based prediction in the application of peer selection.

\subsection{Algorithms}

Thus, two SGD-based decentralized matrix factorization algorithms ${ }^{3}$, denoted by DMFSGD, were designed for dealing with RTT and with ABW, given in Algorithm 1 and in Algorithm 2. Our DMFSGD algorithms have the same architecture as Vivaldi [7] where each node randomly and independently chooses a neighbor set of $k$ nodes as references and randomly probes one of its neighbors at each time. The coordinates of the nodes are initialized with random numbers uniformly distributed between 0 and 1. Empirically, the DMFSGD algorithms are insensitive to the random initialization of the coordinates as well as the random selection of the neighbors.

\section{EXPERIMENTS AND EVALUATIONS}

In this section, we evaluate our DMFSGD algorithms and study the sensitivity to the parameters, the robustness against erroneous measurement and the applicability on peer selection.

\footnotetext{
${ }^{3}$ The matlab implementation of the algorithms is available at http://www.run.montefiore.ulg.ac.be/ liao/DMFSGD.
} 


\subsection{Datasets and Evaluation Criteria}

The evaluations were performed on the following publicly available datasets.

Harvard contains 2, 492,546 dynamic measurements of application-level RTTs, with timestamps, between 226 Azureus clients collected in 4 hours [11].

Meridian contains static RTT measurements between 2500 network nodes obtained from the Meridian project [25].

HP-S3 contains ABW measurements between 459 network nodes collected using the pathchirp tool [26]. As the raw dataset is highly sparse with $55 \%$ missing data, we extracted 231 nodes to construct a dense ABW matrix with $4 \%$ missing entries.

In the simulations, the static measurements in Meridian and HP-S3 are used in random order, whereas the dynamic measurements in Harvard are used in time order according to the timestamps. We built a static matrix for Harvard by extracting the median values of the streams of measurements between each pair of nodes and used it as the ground truth.

The following evaluation criteria [2] were used.

ROC A Receiver Operating Characteristic (ROC) curve is a graphical plot of the true positive rates (TPR) versus the false positive rates (FPR) for a binary classifier as its discrimination threshold is varied.

AUC The AUC is the area under the ROC curve. As the TPR and the FPR range from 0 to 1 , the maximal possible value of AUC is 1 which means a perfect classification. In practice, AUC is smaller than 1 . The closer it is to 1 , the better.

Precision-Recall The precision for a class is the number of true positives divided by the total number of elements labeled as belonging to the positive class, i.e. the sum of true positives and false positives, and the recall for a class is equal to the TPR.

More precisely, the ROC and Precision-Recall curves are obtained by varying a discrimination threshold $\tau_{c}$ when deciding the classes from $\hat{x}_{i j}$ 's. For a given $\tau_{c}, \hat{x}_{i j}$ is turned into 1 if $\hat{x}_{i j}>\tau_{c}$ and into -1 otherwise. Then, the true positive rate, false positive rate and precision for the given $\tau_{c}$ can be computed by comparing $x_{i j}$ 's and the binarized $\hat{x}_{i j}$ 's. The ROC and Precision-Recall curves are then obtained by varying $\tau_{c}$ from $-\infty$ to $+\infty$. These evaluation criteria are interesting and commonly used because they show the prediction accuracies under different $\tau_{c}$ 's.

\subsection{Impact of Parameters}

We demonstrate the impact of the parameters to the accuracy of the prediction, including learning rate $\eta$, regularization coefficient $\lambda$, loss function $l$ : hinge or logistic, rank $r$, neighbor number $k$ and classification threshold $\tau$.

\subsection{1 $\eta$, $\lambda$ and $l$}

$\eta$ controls the step of each update, which has to be small enough to guarantee the convergence of the algorithms while large enough to converge fast. $\lambda$ trades off between the fitting errors and the norms of the solutions, which, similar to $\eta$, has to be small enough to guarantee the overall fitness of the factorization while large enough to avoid overfitting. Besides, the incorporation of the regularization enforces the coordinates of the nodes to have low norms and helps overcome the drifts of the coordinates due to the non-uniqueness of the factorization in eq. 4.

We experimented with different configurations of $\eta$ and $\lambda$ under different loss functions, shown in Figure 3. It can be seen that $\lambda=0.1$ and $\eta=0.1$ work well for all three datasets and that the logistic loss function outperforms the hinge loss function in most cases. Thus, unless stated otherwise, $\lambda=0.1, \eta=0.1$ and the logistic loss function are used by default.

\subsection{2 $r$ and $k$}

Intuitively, $r$ is the number of unknown variables in each coordinate and $k$ is the number of known data used to estimate each coordinate. Clearly, increasing $k$ is equivalent to adding more data thus always helps improve the accuracy. However, a large $k$ also means a higher measurement overhead which may outweigh the benefits of the accurate prediction. On the other hand, increasing $r$ is equivalent to adding more variables to estimate, consuming more data. We found that a pair of relatively small $k$ and $r$ can already provide sufficient classification accuracy, shown in Figure 4(a) and 4 (b), and further increasing $k$ and $r$ is either costly or worthless. Thus, unless stated otherwise, $r=10$ and $k=10,32$ and 10 for Harvard, Meridian and HP-S3 respectively are used by default for all datasets.

\section{$6.2 .3 \tau$}

The classification threshold $\tau$ significantly affects the proportions of the two classes, shown in Table 1, which in turn have some impacts on the prediction accuracy, shown in Figure 4(c). Although in practice $\tau$ is determined according to the requirements of the applications, we nevertheless set $\tau$ to the median value of each dataset by default.

\subsubsection{Discussions}

The default parameter configuration of $\lambda=0.1, \eta=$ $0.1, r=10$ and the logistic loss function is not guaranteed to be optimal for different $k$ 's and $\tau$ 's and on different datasets. However, fine parameter tuning is 
Harvard
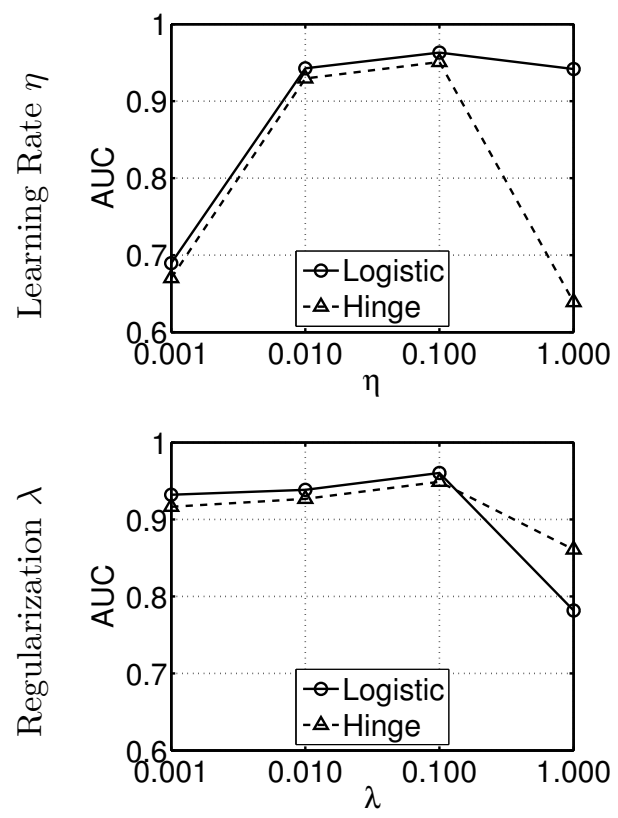

Meridian
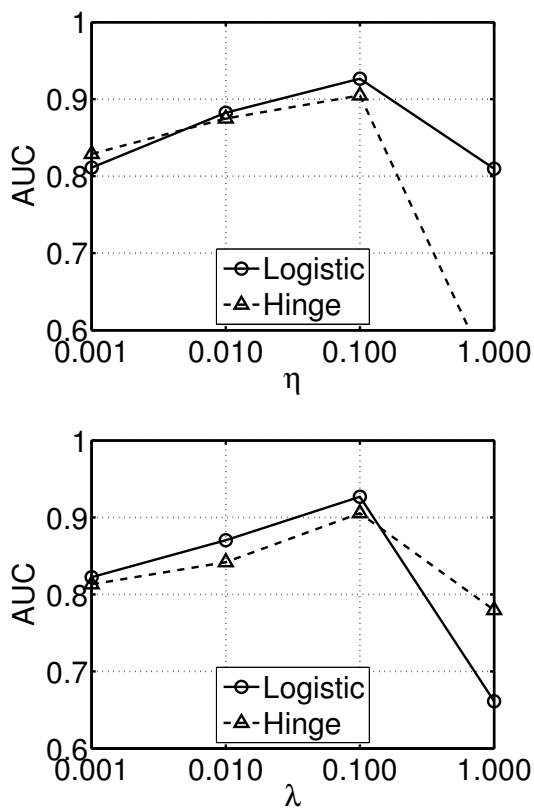

HP-S3
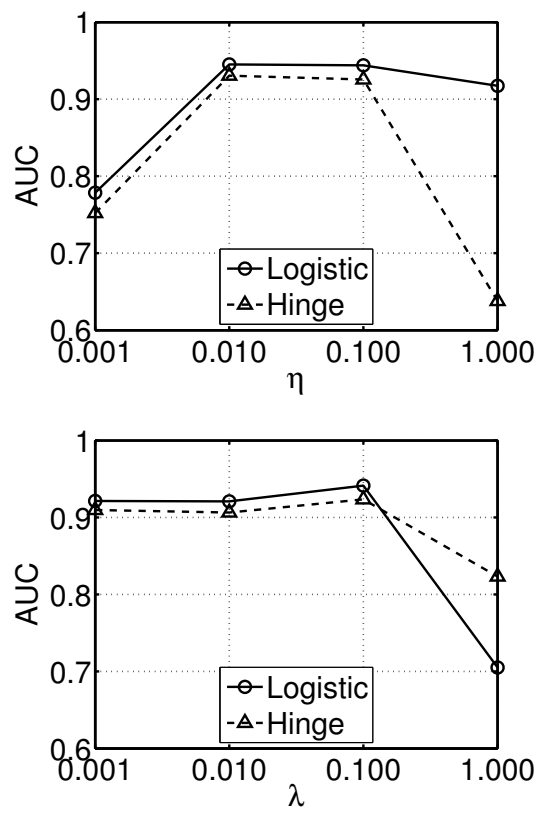

Figure 3: AUCs under different $\eta$ 's and $\lambda$ 's on different datasets. The first row shows the impact of $\eta$ under $\lambda=0.1$ and the second row shows the impact of $\lambda$ under $\eta=0.1 . r=10$ in this figure. $k=10$, 32 and 10 for the Harvard, Meridian and HP-S3 datasets respectively. $\tau$ is set to the median value of each dataset, i.e. $\tau=132 \mathrm{~ms}$ for Harvard, $56 \mathrm{~ms}$ for Meridian and 43Mbps for HP-S3.

Table 1: Impact of $\tau$ on portions of "good" paths in different datasets.

\begin{tabular}{|c|c|c|c|}
\hline \multirow{2}{*}{ "Good"\% } & \multicolumn{3}{|c|}{$\tau$} \\
\cline { 2 - 4 } & $\begin{array}{c}\text { Harvard } \\
(\mathrm{ms})\end{array}$ & $\begin{array}{c}\text { Meridian } \\
(\mathrm{ms})\end{array}$ & $\begin{array}{c}\text { HP-S3 } \\
(\mathrm{Mbps})\end{array}$ \\
\hline $10 \%$ & 27.5 & 19.4 & 88.2 \\
\hline $25 \%$ & 59.9 & 36.2 & 72.2 \\
\hline $50 \%$ & 131.6 & 56.4 & 43.1 \\
\hline $75 \%$ & 249.6 & 88.1 & 14.4 \\
\hline $90 \%$ & 324.2 & 155.2 & 10.4 \\
\hline
\end{tabular}

difficult, if not impossible, for network applications due to the dynamics of the measurements and the decentralized processing where local measurements are processed locally with no central node to gather information.

Figure 5 shows that the recommended default parameters produced fairly accurate results on all three datasets which are largely different from each other. The insensitivity to the parameters is probably because the inputs are binary classes and take values of either 1 or -1 regardless of the actual metric and values. The rightmost plot in Figure 5 illustrates the convergence speeds in terms of the AUC improvements with respect to the average measurement number per node, i.e. the total number of measurements used by all nodes divided by the number of nodes ${ }^{4}$. It can be seen that the DMFSGD algorithms converges fast after each node probes, on average, no more than $20 \times k$ measurements from its $k$ neighbors. Table 2 shows the accuracy rates, i.e., the percentage of the correct predictions, and the confusion matrices, computed by taking the sign of $\hat{x}_{i j}$ 's and then comparing with the corresponding $x_{i j}$ 's. These measures show intuitively the accuracy of our approach under the default parameters.

\subsection{Robustness Against Erroneous Labels}

This section evaluates the robustness of the DMFSGD algorithms against erroneous class labels which arise from inaccurate measurements due to

- inaccurate measurement techniques which particularly affect those paths with metric quantities close to $\tau$;

- network anomaly such as attacks from malicious nodes and sudden traffic bursts which affect every path equally.

\footnotetext{
${ }^{4}$ For P2PSim1740 and Meridian2500, at any time, the number of measurements used by each node is statistically the same for all nodes due to the random selections of the source and the target nodes in the updates. For Harvard226, this number is significantly different for different nodes because the paths were passively probed with uneven frequencies.
} 


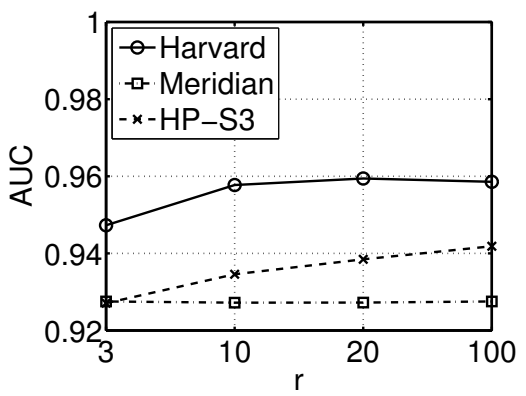

(a) Rank $r$

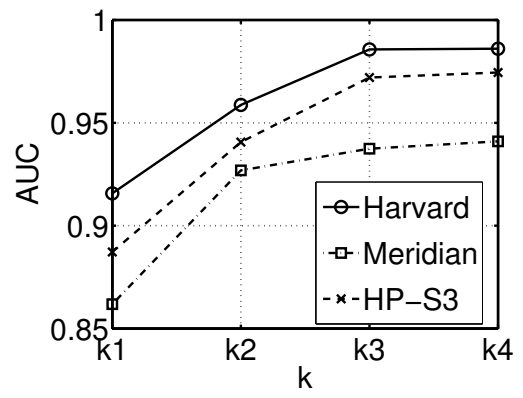

(b) Neighbor number $k$

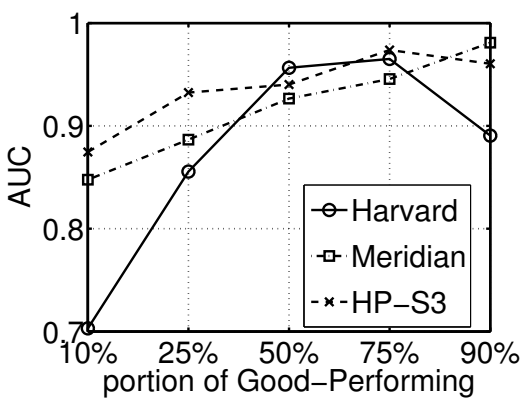

(c) Classification threshold $\tau$

Figure 4: AUCs under different $k$ 's, $r$ 's and $\tau$ 's on different datasets. The left plot shows the impact of $r$ under $k=10$ for Harvard, 32 for Meridian and 10 for HP-S3. The middle plot shows the impact of $k$ under $r=10$ for all datasets. The experimented $k$ 's are $k_{1}=5, k_{2}=10, k_{3}=30$ and $k_{4}=50$ for both Harvard and HP-S3 and $k_{1}=16, k_{2}=32, k_{3}=64$ and $k_{4}=128$ for Meridian. $\tau$ in the left and middle plots is set to the median value of each dataset. The right plot shows the impact of $\tau$ under $r=10$ for all datasets and $k=10$ for Harvard, 32 for Meridian and 10 for HP-S3. The experimented $\tau$ 's for different datasets are listed in Table 1 to generate different portions of "good" paths.

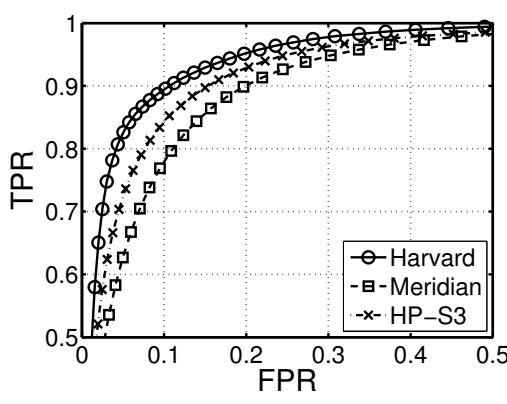

(a) $\mathrm{ROC}$

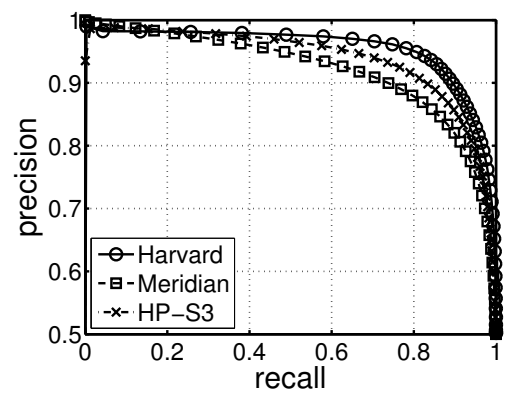

(b) Precision-Recall

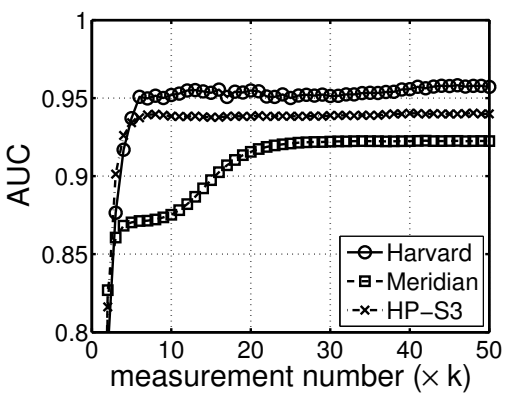

(c) AUC

Figure 5: The accuracy of class-based prediction by DMFSGD on different datasets under the default parameter configuration. The rightmost plot shows the AUC improvements with respect to the average measurement number used by each node.

In particular, we simulated four different types of errors, including

Type 1: flip near $\tau$. Flip randomly, with probability 0.5 , the class labels of the paths with quantities within $[\tau-\delta, \tau+\delta]$.

Type 2: underestimation bias. For $\mathrm{ABW}^{5}$, label erroneously the paths with quantities within $[\tau, \tau+\delta]$ as "bad".

Type 3: flip randomly. For $\mathrm{ABW}^{6}$, choose randomly

\footnotetext{
${ }^{5}$ Most ABW measurement tools such as pathload and pathchirp have a tendency of underestimating ABW [15], whereas such tendency is not reported by RTT measurement tools such as ping.

${ }^{6}$ For most network measurements, "bad" paths are unlikely to be erroneously estimated as "good". However, ABW is probed by the sender but inferred by the target node. Thus, malicious target nodes can purposely respond with flipped class labels.
}

$p \%$ paths and flip their labels.

Type 4: Good-to-Bad. Choose randomly $p \%$ "good" paths and label them as "bad".

We experimented with all four types of errors for the HP-S3 dataset and the errors of Type 1 and 4 for the Harvard and Meridian datasets, shown in Figure 6. Different error levels of $5 \%, 10 \%$ and $15 \%$ erroneous labels were tested by setting different $\delta$ 's and $p$ 's for each type of errors and for each dataset. The values of $\delta$ are shown in Table 3 and $p=5,10$ and 15 .

It can be seen that random errors of "flip randomly" and "Good-to-Bad" have a much larger impact to the classification accuracy than errors of "flip near $\tau$ " and "underestimation bias" that only perturb paths with quantities near $\tau$. The random errors are mostly due to network anomalies. They are therefore rarer and can be addressed by incorporating heuristics such as inferring the class labels using some consensus based on recorded 


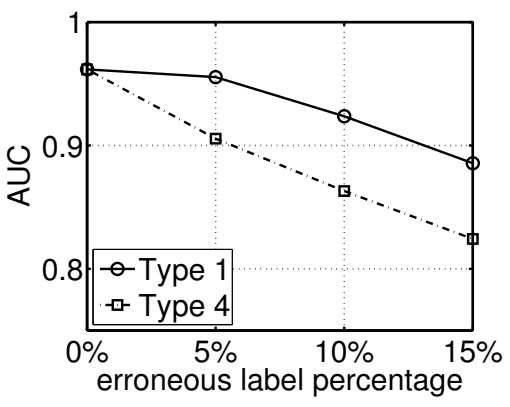

(a) Harvard

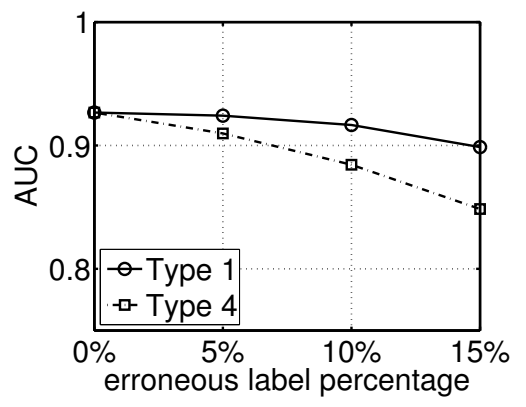

(b) Meridian

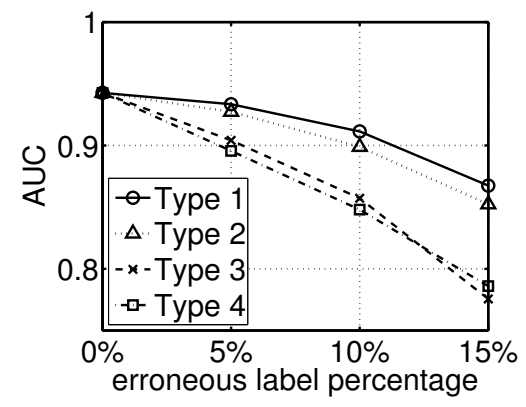

(c) HP-S3

Figure 6: Robustness of class-based prediction against erroneous class labels.

Table 2: Confusion Matrix

\begin{tabular}{|c|c|c|c|c|}
\hline \multirow{4}{*}{ Harvard } & \multirow{2}{*}{\multicolumn{2}{|c|}{ Accuracy $=89.4 \%$}} & \multicolumn{2}{|c|}{ Predicted } \\
\hline & & & "Good" & "Bad" \\
\hline & \multirow{2}{*}{ Actual } & "Good" & $93.6 \%$ & $6.4 \%$ \\
\hline & & "Bad" & $14.7 \%$ & $85.3 \%$ \\
\hline \multirow{4}{*}{ Meridian } & \multirow{2}{*}{\multicolumn{2}{|c|}{ Accuracy $=85.4 \%$}} & \multicolumn{2}{|c|}{ Predicted } \\
\hline & & & "Good" & "Bad" \\
\hline & \multirow{2}{*}{ Actual } & "Good" & $88.5 \%$ & $11.5 \%$ \\
\hline & & "Bad" & $17.8 \%$ & $82.2 \%$ \\
\hline \multirow{4}{*}{ HP-S3 } & \multirow{2}{*}{\multicolumn{2}{|c|}{ Accuracy $=87.3 \%$}} & \multicolumn{2}{|c|}{ Predicted } \\
\hline & & & "Good" & "Bad" \\
\hline & \multirow{2}{*}{ Actual } & "Good" & $93.5 \%$ & $6.5 \%$ \\
\hline & & "Bad" & $18.9 \%$ & $81.1 \%$ \\
\hline
\end{tabular}

Table 3: The values of $\delta$ that lead to certain error levels in Figure 6.

\begin{tabular}{|c|c|c|c|c|}
\hline \multirow{2}{*}{ error\% } & \multicolumn{4}{|c|}{$\delta$} \\
\cline { 2 - 5 } & Harvard (ms) & Meridian (ms) & \multicolumn{2}{|c|}{ HP-S3 (Mbps) } \\
\cline { 2 - 5 } & Type 1 & Type 1 & Type 1 & Type 2 \\
\hline $5 \%$ & 24.4 & 5.2 & 3.2 & 2.9 \\
\hline $10 \%$ & 41.5 & 10.2 & 6.7 & 5.7 \\
\hline $15 \%$ & 54.7 & 14.8 & 13.2 & 10.0 \\
\hline
\end{tabular}

historical measurements. The errors of "flip near $\tau$ " and "underestimation bias" are mostly due to the inaccuracies of the measurement tools and can generally be reduced at the cost of more probe traffics, which is less necessary due to their limited impact.

\subsection{Peer Selection: Optimality VS. Satisfaction}

For many Internet applications such as peer-to-peer downloading and streaming, the exploitation of end-toend network performance is to find for each node a satisfactory node to interact with from a number of candidates. Motivated by this demand, we demonstrate how peer selection can benefit from the prediction of network performance classes. We also compare class- based and quantity-based prediction on peer selection using the same DMFSGD algorithms with the quantitybased prediction adopting the $L_{2}$ loss function and the corresponding gradient functions in eqs. 18 and 19.

To this end, we let each node randomly select a set of peers from all connected nodes. The nodes in the peer set are forced to be different from those in the neighbor set. For class-based prediction, we need to select the peer in the peer set which is the most likely to be "good". This is done by selecting the peer with the largest predicted value, i.e., for node $i$,

$$
j_{p}=\arg \max _{j \in \operatorname{PeerSet}(i)} \hat{x}_{i j} .
$$

Note that we directly use the output of $\hat{x}_{i j}=u_{i} v_{j}^{T}$ without taking its sign or thresholding it by $\tau_{c}$. For quantitybased prediction, peer selection is done by choosing for each node the predicted best-performing node in the peer set, i.e., the smallest $\hat{x}_{i j}$ for RTT and the largest $\hat{x}_{i j}$ for ABW. To demonstrate the impact of erroneous labels to peer selection, we simulated 10\% "flip near $\tau$ " and 5\% "Good-to-Bad" errors, leading to overall $15 \%$ erroneous labels for all datasets. The random peer selection is used as a baseline method for comparison.

The commonly-used evaluation criterion for peer selection is the stretch [27], defined as

$$
s_{i}=\frac{x_{i \bullet}}{x_{i \circ}},
$$

where $\bullet$ is the id of the selected peer, $\circ$ is that of the true best-performing peer in the peer set of node $i$ and $x_{i *}$ is the measured quantity of some performance metric. $s_{i}$ is larger than 1 for RTT and smaller than 1 for ABW. The closer $s_{i}$ is to 1 , the better.

The stretch reflects the optimality of peer selection, shown in the first row of Figure 7. As expected, peer selection based on both class-based and quantity-based prediction outperforms random peer selection and the best optimality is achieved by using quantity-based prediction. Indeed, class-based prediction seeks to provide satisfactory instead of optimal services. To demonstrate this, we plot the average percentage of unsatisfied 
Harvard
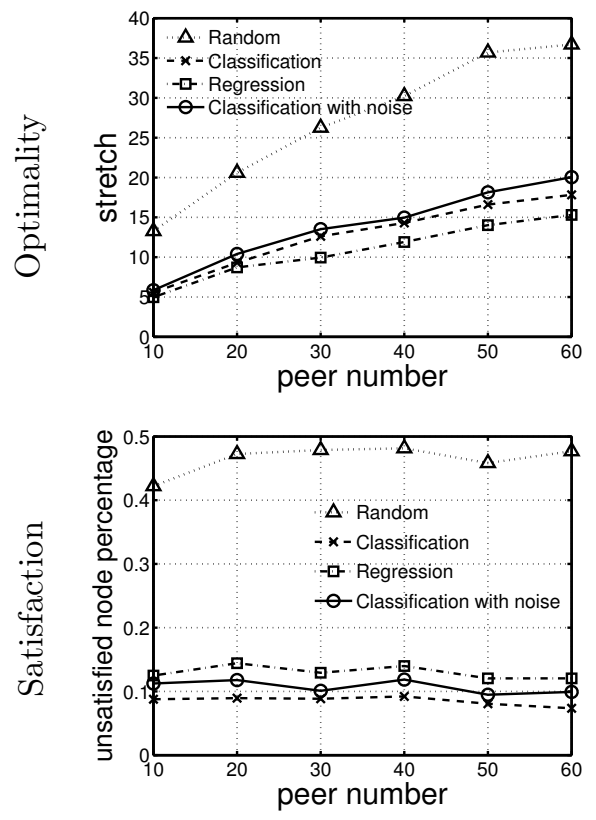

Meridian
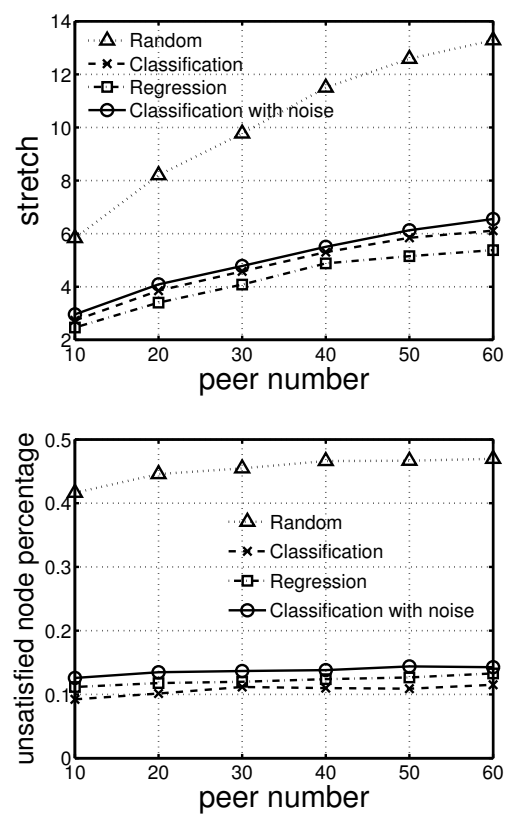

HP-S3
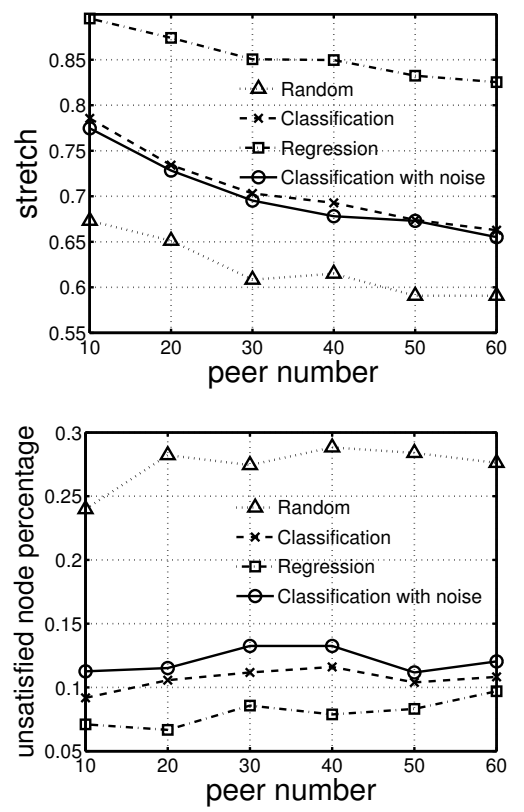

Figure 7: Peer selection with various numbers of peers in the peer set of each node. The top row shows the optimality of the peer selection in terms of the average stretch, and the bottom row shows the satisfaction in terms of the average percentage of unsatisfied nodes, defined as the nodes that select wrongly "bad" peers when there exist "good" peers in the peer sets. The nodes with a peer set of all "bad" peers are excluded from the calculation as no satisfactory peers can be selected.

nodes, defined as the nodes that select wrongly "bad" peers when there are "good" peers available in the peer sets, shown in the second row of Figure 7 . It can be seen that in terms of satisfaction, class-based prediction is sufficient to provide satisfactory services with about on average $10 \%$ unsatisfied nodes and as large as $15 \%$ erroneous labels only degrade the performance of peer selection by less than $5 \%$ for all datasets.

Note that quantity-based prediction achieved on HPS3 a marginally better performance, less than $5 \%$, on the percentage of unsatisfied nodes than class-based prediction, however at the cost of much more measurement overheads in order to get precise $\mathrm{ABW}$ values. It should also be noted that always selecting bestconnected nodes doesn't make efficient use of the overall capacity of the networks and may cause congestions and overloading to those nodes due to their popularity especially in the beginning of the services [6].

\section{CONCLUSIONS AND FUTURE WORKS}

This paper presents a novel approach to predicting end-to-end network performance classes. The success of the approach roots both in the qualitative representation of network performance and in the exploration of the low-rank nature of performance matrices. The former lowers greatly the measurement cost and enables a unified treatment of various performance metrics, while the latter enjoys the recent advances in matrix completion techniques, particularly the stochastic optimization which makes a fully decentralized processing possible. Our so-called Decentralized Matrix Factorization by Stochastic Gradient Descent (DMFSGD) algorithms are highly scalable, able to deal with dynamic measurements in large-scale networks, while robust to erroneous measurements, which is the first of its kind.

While we focus here on binary classification, our framework could be extended to the prediction of more than two performance classes, i.e., multiclass classification, which we would like to study in the near future. The evaluation on peer selection shows the usability of our approach in real applications such as peer-to-peer file sharing and content distribution systems. In the future, we would also like to deploy one such system to test whether these applications can benefit from the simplicity, the flexibility, and the superiority of our approach.

\section{Acknowledgments}

We thank Venugopalan Ramasubramanian for providing us the HP-S3 dataset. We thank Augustin Chaintreau for shepherding this paper and the anonymous reviewers for their helpful feedback. This work was partially supported by the EU under project FP7-Fire 
ECODE, by the European Network of Excellence PASCAL2 and by the Belgian network DYSCO (Dynamical Systems, Control, and Optimization), funded by the Interuniversity Attraction Poles Programme, initiated by the Belgian State, Science Policy Office. The scientific responsibility rests with its authors.

\section{REFERENCES}

[1] D. Bertsekas. Nonlinear programming. Athena Scientific, 1999.

[2] C. M. Bishop. Pattern Recognition and Machine Learning. Springer-Verlag, New York, NJ, USA, 2006.

[3] L. Bottou. Online algorithms and stochastic approximations. In D. Saad, editor, Online Learning and Neural Networks. Cambridge University Press, 1998.

[4] A. M. Buchanan and A. W. Fitzgibbon. Damped newton algorithms for matrix factorization with missing data. In Computer Vision and Pattern Recognition, 2005.

[5] E. J. Candès and Y. Plan. Matrix completion with noise. Proc. of the IEEE, 98(6), 2010.

[6] M. Crovella and B. Krishnamurthy. Internet Measurement: Infrastructure, Traffic and Applications. John Wiley \& Sons, Inc., New York, NY, USA, 2006.

[7] F. Dabek, R. Cox, F. Kaashoek, and R. Morris. Vivaldi: A decentralized network coordinate system. In Proc. of ACM SIGCOMM, Portland, OR, USA, Aug. 2004.

[8] B. Donnet, B. Gueye, and M. A. Kaafar. A survey on network coordinates systems, design, and security. IEEE Communication Surveys and Tutorial, 12(4):488-503, 2010.

[9] Google TV. http://www.google.com/tv/.

[10] M. Jain and C. Dovrolis. End-to-end available bandwidth: measurement methodology, dynamics, and relation with TCP throughput. IEEE/ACM Transactions on Networking, 11:537-549, Aug. 2003.

[11] J. Ledlie, P. Gardner, and M. I. Seltzer. Network coordinates in the wild. In Proc. of USENIX NSDI, Cambridge, Apr. 2007.

[12] Y. Liao, P. Geurts, and G. Leduc. Network distance prediction based on decentralized matrix factorization. In Proc. of IFIP Networking Conference, Chennai, India, May 2010.

[13] Y. Mao, L. Saul, and J. M. Smith. IDES: An Internet distance estimation service for large networks. IEEE Journal On Selected Areas in Communications, 24(12):2273-2284, Dec. 2006.

[14] T. S. E. Ng and H. Zhang. Predicting Internet network distance with coordinates-based approaches. In Proc. of IEEE INFOCOM, New
York, NY, USA, June 2002.

[15] R. S. Prasad, M. Murray, C. Dovrolis, K. Claffy, R. Prasad, and C. D. Georgia. Bandwidth estimation: Metrics, measurement techniques, and tools. IEEE Network, 17:27-35, 2003.

[16] V. Ramasubramanian, D. Malkhi, F. Kuhn, M. Balakrishnan, A. Gupta, and A. Akella. On the treeness of Internet latency and bandwidth. In ACM SIGMETRICS / Performance, Seattle, WA, USA, June 2009.

[17] S. Ratnasamy, P. Francis, M. Handley, R. Karp, and S. Shenker. A scalable content-addressable network. In Proc. of ACM SIGCOMM, San Diego, CA, USA, Aug. 2001.

[18] S. Ratnasamy, M. Handley, R. Karp, and S. Shenker. Topologically-aware overlay construction and server selection. In Proc. of IEEE INFOCOM, June 2002.

[19] V. J. Ribeiro, R. H. Riedi, R. G. Baraniuk, J. Navratil, and L. Cottrell. pathchirp: Efficient available bandwidth estimation for network paths. In Proc. of the Passive and Active Measurement, 2003.

[20] I. Rish and G. Tesauro. Estimating end-to-end performance by collaborative prediction with active sampling. In Integrated Network Management, pages 294-303, May 2007.

[21] A. Shriram, M. Murray, Y. Hyun, N. Brownlee, A. Broido, M. Fomenkov, and K. Claffy. Comparison of public end-to-end bandwidth estimation tools on high-speed links. In Proc. of the Passive and Active Measurement, pages 306-320, 2005.

[22] N. Srebro, J. D. M. Rennie, and T. S. Jaakola. Maximum-margin matrix factorization. In Advances in Neural Information Processing Systems, pages 1329-1336, 2005.

[23] L. Tang and M. Crovella. Virtual landmarks for the Internet. In Proc. of ACM/SIGCOMM Internet Measurement Conference, Oct. 2003.

[24] Vuze Bittorrent. http://www. vuze.com/.

[25] B. Wong, A. Slivkins, and E. Sirer. Meridian: A lightweight network location service without virtual coordinates. In Proc. of ACM SIGCOMM, Aug. 2005.

[26] P. Yalagandula, P. Sharma, S. Banerjee, S. Basu, and S.-J. Lee. S3: a scalable sensing service for monitoring large networked systems. In Proc. of the 2006 SIGCOMM workshop on Internet network management, pages 71-76, 2006.

[27] R. Zhang, C. Tang, Y. C. Hu, S. Fahmy, and $\mathrm{X}$. Lin. Impact of the inaccuracy of distance prediction algorithms on Internet applications: an analytical and comparative study. In Proc. of IEEE INFOCOM, Barcelona, Spain, Apr. 2006. 